\title{
Optimization of selective assembly and adaptive manufacturing by means of cyber- physical system based matching
}

\author{
Gisela Lanza (2), Benjamin Haefner*, Alexandra Kraemer \\ wbk Institute of Production Science, Karlsruhe Institute of Technology (KIT), Kaiserstr. 12, 76131 Karlsruhe, Germany
}

In high-tech production, companies often deal with the manufacturing of assemblies with quality requirements close to the technological limits. Selective and adaptive production systems are means to cope with this challenge. In this context new measurement technologies and IT-systems offer the opportunity to generate and use real-time quality data along the process chain and to control the production system adaptively. In this article, a holistic matching approach to optimize the performance of selective and adaptive assembly systems is presented and its industrial application within an automotive electric drive assembly is demonstrated.

Assembly; Quality assurance; Tolerancing

\section{Motivation}

In today's high-tech industries such as micro [1] or automotive production (e.g. of crankshafts, pistons or electric drive rotors) $[2,3,4]$, the requirements to the product quality in more and more cases approach the technological limits of the potential manufacturing processes. In particular, for assemblies with very small tolerances, the manufacturing processes of the components are either not capable of guaranteeing the required precision or only at very high cost.

Yet, the ongoing evolution of information and communication technologies (ICT), characterized by increasing computational capacity, functionalized sensor technology and ubiquitous network structures, is a means to cope with this challenge. By their integration into production facilities so called cyberphysical production systems can be created [5]. These enable intelligent interactions of the physical elements as well as adaptive and decentralized control of the system. This development commonly is referred to as "Industrie 4.0" associated with the understanding of a fourth industrial revolution.

As an enabler ICT provide great potential to be exploited for the aforementioned challenge of the production of high precision products at reasonable cost and to compensate limited manufacturing capabilities. By the conduction of inline measurements within the process chain, real-time information about quality characteristics at all critical production stages can be gathered. It is expected that cost-efficient inline sensors will be available as a commodity for many purposes in production in the future. Using the sensor data, real-time control in production can be facilitated.

The characteristics of the production system, in general, can be altered at three degrees of freedom:

- Selective assembly: matching of compatible low-precision components to achieve an assembly of high precision

- Adaptive manufacturing: manipulation of manufacturing parameters to build suitable components for assembly

- Product co-design: adjustment of the component design to
Within the scope of this article only selective assembly and adaptive manufacturing are considered. Both approaches have in common that the complexity of the limited manufacturing processes is mitigated to an increased structural complexity of the production system [4]. In this article, a new approach of selective assembly and adaptive manufacturing is presented in which the configuration of the production system is optimized in real-time by means of inline quality measurements.

\section{State of the art}

Selective assembly has been investigated by many researchers. Most approaches on selective assembly are based on the idea of partitioning components into classes of equal width. Regarding this issue, various statistical methods have been developed to find optimal binning strategies, e.g. by Mease et al. [2], Matsuura et al. [3] or Kannan et al. [6]. Fang et al. [7] propose methods based on classes with equal probabilities. Kannan et al. [8], Babu et al. [9], Asha et al. [10] and Kumar et al. [11] present different optimization algorithms for the matching of tolerance classes based on particle-swarm-optimization, artificial immune systems or genetic algorithms. Raj et al. [12] introduce a genetic algorithm to optimally mate the components within a batch. Some other approaches also incorporate adaptive manufacturing in selective assembly. Akansel et al. [13] present an approach in which the manufacturing settings of one of the components can dynamically be adjusted. Iyama et al. [14] developed an optimal corrective assembly approach including reprocessing of components. Reprocessing and optimal fitting algorithms have been studied at wbk Institute of Production Science in the complex case of space frame structures [15]. Schmitt et al. [16] developed a selfoptimization method for the adaption of manufacturing parameters in a process chain. Arai [17], Codellani et al. [3] and Kayasa et al. [18] developed process models to analyse various effects of production systems with selective assembly and adaptive manufacturing such as machine reliability or work in progress. While most of the approaches are restricted to the matching of only two components, some also deal with three ones, e.g. [8], [9], [12]. At wbk Institute of Production Science, 
magnets in rotor assembly without partitioning into classes is studied [19]. Utilizing ICT, a concept for RFID-based, situational shop floor control was recently developed by Engelhardt et al. [20], yet, not dealing with the challenges of selective assembly.

In summary, it can be found that currently there is no research approach dealing with selective and adaptive production systems which considers the following aspects in detail:

- Methodology for the real-time optimization of a selective and adaptive production system with inline quality measurements

- Optimization algorithm for individual matching of components based on their specific measurement values in real-time

- Selective assembly and adaptive manufacturing for assemblies with more than three components

\section{Research approach}

To overcome the aforementioned limitations, in this article a research approach is presented which enables an adaption of a production system based on inline quality measurements at relevant intermediate steps of the process chain in real time.

\subsection{General model of the cyber-physical production system}

The model of this research approach, illustrated in Fig. 1, focuses on a production system consisting of two process chains of manufacturing processes $P_{1}$ and $P_{2}$, whose final components are joined in an assembly station $A$. The model incorporates that an arbitrary number of the component types 1 and 2 has to be assembled for the final product. Without loss of generality the process chains of the two components can be considered as two single manufacturing processes. Cost-efficient inline metrology, e.g. using optical sensors, enables to measure the quality of each component and the final product at inline inspection stations $Q_{1}$, $Q_{2}$ and $Q_{\text {final }}$. Furthermore, the cyber-physical production system comprises an adaptive controller as well as a knowledge base. After the inline inspection stations $Q_{1}$ and $Q_{2}$ the inventory $I_{1}$ and $I_{2}$ are located, in which the two component types can be stored. It is assumed without loss of generality that the process capability of process $P_{2}$ is equal or higher than that of $P_{1}$. Thus an adaption of the manufacturing processes can be realized by a shift $\theta_{2}$ of the nominal value of the resp. parameter $p_{2}$ of process $P_{2}$.

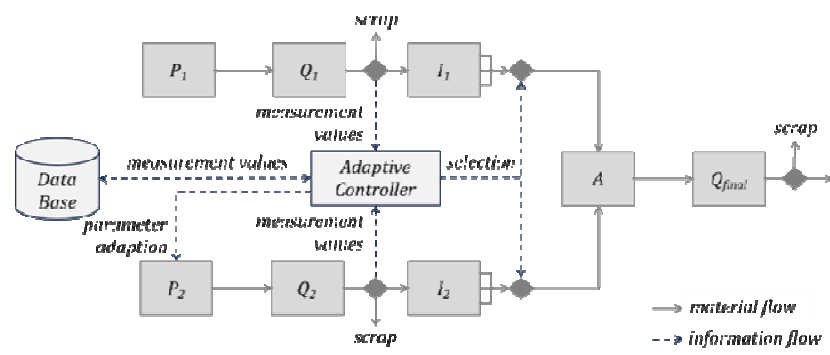

Figure 1. Model of the considered cyber-physical production system.

\subsection{Adaptive control of the cyber-physical production system}

The adaptive control of the cyber-physical production system is realized by virtually mirroring the real production system in a discrete event simulation to represent its cause-effect relationships as realistically as possible. By means of a data interface the measurement values of the inspection stations can be imported to the control software. After the execution of optimization algorithms in the control software, the resulting control commands are forwarded to the real production system in real-time. The adaptive controller is capable of dynamically and autonomously optimizing the production system by means of simulating and evaluating potential control options simultaneously. The optimization is based on genetic algorithms. Thus, at each point in time the best configuration with regard to selective assembly and adaptive manufacturing can be selected. The optimization of the controller is divided into two time horizons with structural and real-time control cycles (Fig. 2).

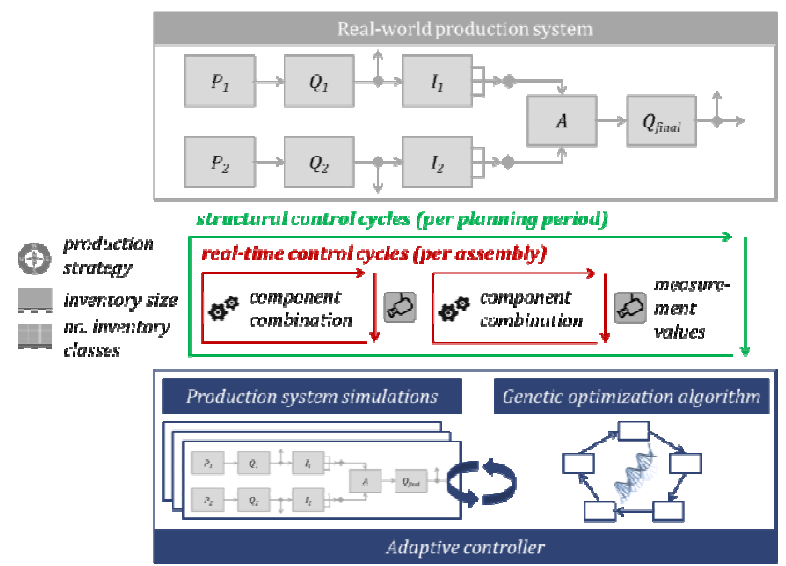

Figure 2. Structure of the adaptive control.

\subsection{Optimization algorithm of structural control cycles}

In the structural control cycles of each planning period $t$ the configurations of potential, predefined production strategies $s$ are evaluated and the optimal one is selected. For this, the result of the real production system in period $t-1$ with its selected strategy $s$ is compared to simulations according to the other strategies based on the quality measurements in period $t-1$. For each production strategy $s$ the inventory sizes $n_{s, 1}$ and $n_{s, 2}$ and the numbers of classes $c_{s, 1}$ and $c_{s, 2}$ are optimized, being the relevant variables in selective assembly in addition to the production strategy. The overall objective in this optimization approach is the reduction of the total cost $C$ of the production system, as in most cases this is the crucial evaluation criterion for its performance. Thus, for the optimization problems in the structural control cycles the objective function is defined as:

$\min _{\Theta} C=\min _{s}\left\{\sum_{i=1}^{N_{e f f}(\Theta)} C_{P}(\Theta)+C_{Q}(\Theta)+C_{I}(\Theta)\right\}$

where $C_{P}$ represents the production cost, $C_{Q}$ the quality cost and $C_{I}$ the inventory cost accumulating within the required period to produce a defined amount $N_{\text {eff }}$ of good assemblies within the tolerance limits. $C_{P}$ includes the cost for material, machines and personnel of all good assemblies. $C_{Q}$ is defined as the scrap cost of the defective parts, which is given by their accumulated added values at the scrapping points within the process chain. $C_{I}$ refers to the cost for storing the components of type 1 and 2 in the inventories. The decision variables $\Theta$ of the optimization problem are defined by:

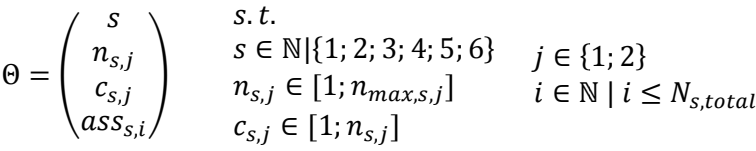

where $s$ is the production strategy, $n_{s, j}$ are the inventory sizes and $c_{s, j}$ the number of classes, into which these are divided. The inventories are restricted by the maximum technical limits $n_{\max , s, i}$ $a s s_{s, i}$ are the realizations of the $N_{s, t o t a l}$ assemblies totally mounted by strategy $s$. There are six production strategies $s$ considered: 
(1) conventional (no selective assembly/adaptive manufacturing)

(2) selective assembly (with classes)

(3) individual assembly (no classes)

(4) selective assembly (with classes) and adaptive manufacturing

(5) individual assembly (no classes) and adaptive manufacturing

(6) individual manufacturing

In strategy (1) the product is assembled conventionally without any type of selective assembly and adaptive manufacturing. Strategy (2) represents selective assembly, where each component after its measurement is sorted into one of the $c_{s, 1}$ resp. $c_{s, 2}$ classes of equal width. If a class is occupied, the component has to be discarded. The optimal component combination for each assembly is determined within the real-time control cycles.

Strategy (3) is an innovative assembly approach called individual assembly without partitioning into inventory classes. Exploiting the opportunities of the inline sensor technology, for each assembly in the real-time control cycle an optimal combination of components currently available in the inventories is selected based on the individual components' measurement values. Thus, in contrast to strategy (2) the full degree of information of the measurements can be used.

In strategy (4) and (5) selective and individual assembly are combined with adaptive manufacturing. In each period $t$ of the structural cycles before starting the optimization, a parameter shift $\theta_{2, t}$ of process $P_{2}$ is calculated based on the means of the measurement values $\mu_{2, t-1}$ and $\mu_{2, t-2}$ in the previous periods such that $\Delta \mu_{t-1}\left(\theta_{2, t}\right)=\mu 2, t-2-\mu 2, t-1$.

If there is the possibility in a production system that for each assembly the more capable process $P_{2}$ can be executed after each component measurement at $Q_{1}$, parameter $p_{2}$ can be individually set for the component manufacturing at $P_{2}$, which is subject to the strategy of individual manufacturing (strategy 6).

To determine the realizations of the assemblies $a s s_{s, i}$ in the strategies (2) - (5) the real-time control cycles are executed. The optimization problems of the structural control cycles as well as the real-time control cycles are solved by means of genetic algorithms, heuristically identifying an optimal solution based on repeated recombination, mutation and selection.

\subsection{Optimization algorithms of real-time control cycles}

In the real-time control cycles for the respective assembly $a s s_{s, i}$ an optimal combination of the components currently available in the inventories is determined. $\mathrm{CO}_{1, k}$ resp. $\mathrm{CO}_{2, l}$ are the selected $N_{1}$ resp. $N_{2}$ components of type 1 and 2 for assembly $a s s_{s, i}$ :

$$
\operatorname{ass}_{S, i}=\left\{\mathrm{Co}_{1,1} ; \mathrm{Co}_{1, k} ; \ldots ; \mathrm{Co}_{1, \mathrm{~N}_{1}} ; \mathrm{CO}_{2,1} ; \mathrm{CO}_{2, l} ; \ldots ; \mathrm{CO}_{2, \mathrm{~N}_{2}}\right\}
$$

For the strategies $s$ with individual assembly the objective function is defined such that component combinations close to the tolerance limits, $L S L_{a}$ resp. $U S L_{a}$, of the assembly clearance $a$ are chosen to prevent the inventory running empty of good parts. This approach only refers to one assembly clearance quantity $a$ :

$$
\begin{aligned}
& \min _{c o_{1, k}, c o_{2, l}}\left\{\min \left[f\left(c o_{1, k}, c o_{2, l}\right)-L S L_{a} ; U S L_{a}-f\left(\mathrm{co}_{1, k}, c o_{2, l}\right)\right]\right\} \\
& \text { s.t. } \quad L S L_{a} \leq f\left(c o_{1, k}, c o_{2, l}\right) \leq U S L_{a}
\end{aligned}
$$

Utilizing ICT effectively, in individual assembly (strategies (3) and (5)) the clearance can be calculated using the measurement values $m\left(\mathrm{Co}_{1, k}\right)$ and $m\left(\mathrm{CO}_{2, l}\right)$, thus $f\left(\mathrm{CO}_{1, k}, \mathrm{CO}_{2, l}\right)=f\left(m\left(\mathrm{Co}_{1, k}\right), m\left(\mathrm{Co}_{2, l}\right)\right)$.

Similarly, this method is also applied for the selective assembly strategies with inventory classes (strategies (2) and (4)). Yet, the clearance is calculated based on the class means $m_{c}\left(\mathrm{CO}_{1, k_{0}}\right)$ and
$m_{c}\left(\mathrm{CO}_{2,1}\right)$ of the inventory classes, in which the components are located, $f\left(\mathrm{Co}_{1, k}, \mathrm{CO}_{2, l}\right)=f\left(m_{c}\left(\mathrm{Co}_{1, k}\right), m_{c}\left(\mathrm{Co}_{2, l}\right)\right)$.

\section{Case study}

\subsection{Industrial background}

This research approach has been studied in a use case regarding the stator production of an automotive transmissionintegrated hybrid electric drive. The stator has a single pole design, containing 30 single teeth, which are assembled with a stator housing (Fig. 3).

The manufacturing of the single teeth is very complex. First electrical steel sheets with an insulation coating are stamped and stacked to single teeth by backed varnish. Then, after a baking operation, a plastic insulating layer is added by injection moulding. Finally, the last steps are the winding of the teeth with electrical wires and their impregnation by soaking them in insulating varnish. The stator housing is externally manufactured by a turning operation. For assembly 30 teeth are radially aligned in a circle. By applying a defined radial preload to the teeth, they are pressed into the stator housing. The assembly requires an interference fit of the outer teeth circle diameter over the inner housing diameter within a clearance range of $0.50 \mathrm{~mm}$. The mounting, currently, is realized without any selective assembly. Yet, there is the possibility to measure the geometry of each tooth and the housing before assembly by optical-tactile CMMs. While the housing diameter can be directly measured, the teeth circle diameter depends on the geometry of all 30 teeth. For simplification of the metrological task the secant $m$ of the teeth was identified as characteristic quantity (Fig. 3).
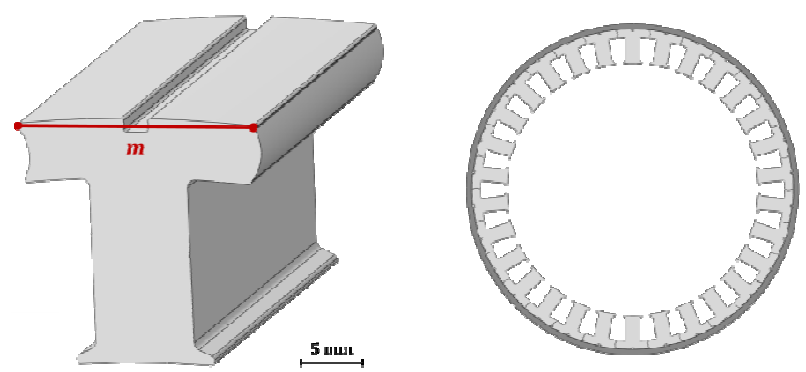

Figure 3. Single tooth with characteristic secant $m$ (left) and stator consisting of stator housing and 30 single teeth (right).

\subsection{Results}

The presented model was implemented in the software Plant Simulation 11 by Siemens PLM Software, incorporating the genetic optimization algorithms of the adaptive control. Based on the characteristics of the production system stated in Table 1 and measurement values of the components, the model of the adaptively controlled cyber-physical production system was verified and validated. The duration of a structural control cycle was defined by the production volume of 5000 stators per period. In this case study the adaptive control of the cyber-physical production system was applied for the duration of four structural control cycles. Fig. 4 summarizes the results showing the average total cost $C_{\text {total }}$ per assembly at each day.

Table 1 Parameters of the production system

\begin{tabular}{llll}
\hline Parameter & Teeth & Housing & Assembly \\
\hline Production cost (EUR/pc.) & 0,55 & 45 & 5 \\
Quality cost (EUR/pc. of scrap) & 0,55 & 45 & 66,50 \\
Inventory cost (EUR/inventory space) & 5 & 12 & 16 \\
\hline
\end{tabular}


Table 2 Optimization results of the adaptive control for all production strategies for period $t=2$ based on the quality measurements of period $t=1$

\begin{tabular}{|c|c|c|c|c|c|c|}
\hline Quantity & (1) Conventional & (2) SA & (3) IA & (4) SA with AM & (5) IA with AM & (6) IM \\
\hline Total cost (EUR) & 543,129 & 420,782 & 403,323 & 349,928 & 340,067 & 338,513 \\
\hline Quality cost (EUR) & 209,048 & 71,976 & 59,724 & 6,214 & 5,808 & 5,416 \\
\hline Inventory cost (EUR) & 473 & 15,953 & 10,913 & 10,913 & 1,153 & 473 \\
\hline Scrap rate & $37 \%$ & $16 \%$ & $14 \%$ & $0 \%$ & $0 \%$ & $0 \%$ \\
\hline No. inventory spaces teeth & 30 & 1320 & 900 & 900 & 120 & 30 \\
\hline No. classes of teeth inventory & 1 & 127 & 900 & 90 & 120 & 1 \\
\hline Parameter for housing diameter (mm) & 289.75 & 289.75 & 289.75 & 290.35 & 290.39 & individual \\
\hline
\end{tabular}

SA = Selective Assembly, IA = Individual Assembly, AM = Adaptive Manufacturing, IM = Individual Manufacturing.

In period $t=1$ production was set to conventional stator assembly (strategy (1)). Based on the measurement values in $t=1$ the controller simulated and optimized the decision variables of the strategies (2) - (6) for period $t=2$. The results of this optimization cycle are summarized in Table 2. As the housing is manufactured externally, individual manufacturing, however, had been defined as an invalid option for period $t=2$ and $t=3$. Furthermore, for period $t=2$ changing of the machining parameters $\theta_{2, t=2}$ in the turning operation of the inner housing diameter was not considered. Thus, individual assembly succeeded as the best available option (strategy (3)) reducing the total cost by $26 \%$. Compared to selective assembly, in particular, this strategy showed lower inventory cost due to a lower amount of inventory spaces and lower quality cost due to a lower scrap rate. Hence, in period $t=2$ this strategy was applied for the assembly. For each stator the best combination of parts currently available in the inventories were selected. Similarly, at the end of period $t=2$ the optimal configuration for $t=3$ was identified. At this point, a change of the machining parameters for the housing diameter was considered valid. In this case individual assembly with adaptive manufacturing was identified to be the best option (strategy (4)), resulting in a cost reduction of $37 \%$ compared to $t=1$. Finally, as in $t=4$ individual manufacturing was possible, strategy (6) was considered in the optimization after $t=3$ and showed the best result with a decrease of the total cost of $38 \%$ compared to $t=1$.

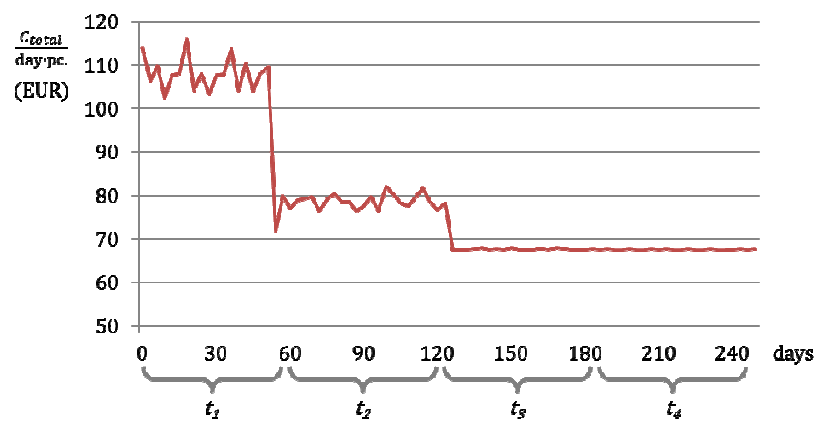

Figure 4. Dynamic results for the adaptive control of the production.

\section{Summary and outlook}

In this article a method for the real-time optimization of selective and adaptive production systems based on ICT-enabled quality measurements is presented. Within this a new optimization algorithm for the individual matching of components in real-time based on their specific measurement values is shown. The approach is applied to a use case of stator assembly for an electrical drive consisting of 30 single teeth and a housing. Further research will extend the model, e.g. with regard to more than two component types and more assembly stations, multicriteria objective systems and robustness studies.

\section{Acknowledgments}

The presented method is part of a research project that is funded by the German Federal Ministry of Education and Research (BMBF) within the Framework Concept "Research for Tomorrow's Production" (funding number 02PJ2415) and managed by the Project Management Agency Karlsruhe (PTKA). The authors are responsible for the contents of this publication. The authors would like to thank all project partners for their support. Moreover, the authors would like to thank, Mr. Florian Lenhoff, Mr. Max Quiring, Mr. Vincent Holleaux and Mrs. Stefanie Minges for their contribution to this article.

\section{References}

[1] Loechte C, Kayasa J, Herrmann C, Raatz A (2012) Methods for Implementing Compensation Strategies in Micro Production Systems Supported by a Simulation Approach. Precision Assembly Technologies and Systems 371:118-125.

[2] Mease D, Nair, VN, Sudjianto A (2004) Selective Assembly in Manufacturing: Statistical Issues and Optimal Binning Strategies. Technometrics 46(2):165-175

[3] Matsuura S, Shinozaki N (2011) Optimal Process Design in Selective Assembly when Components with Smaller Variance are Manufactured at Three Shifted Means. International Journal of Production Research 49(3):869-882.

[4] Colledani M, Ebrahimi D, Tolio T (2014) Integrated quality and production logistics modelling for the design of selective and adaptive assembly systems. CIRP Annals - Manufacturing Technology 62(1):5-8.

[5] Monostori L (2014) Cyber-physical production systems: Roots, expectations and R\&D challenges. Procedia CIRP 17: 9-13.

[6] Kannan SM, Jayabalan V (2002) A new grouping method for minimizing the surplus parts in selective assembly. Quality Engineering 14(1):67-75

[7] Fang XD, Zhang Y (1995) A New Algorithm for Minimizing the Surplus Parts in Selective Assembly. Computers and Industrial Engineering 28(2):341-350.

[8] Kannan SM, Sivasubramanian R, Jayabalan V (2009) Particle swarm optimization for minimizing assembly variation in selective assembly. Int J Adv Manuf Technol 42:793-803.

[9] Babu JR, Asha A (2014) Tolerance modelling in selective assembly for minimizing linear assembly tolerance variation and assembly cost by using Taguchi and AIS algorithm. Int J Adv Manuf Technol 75:869-881.

[10] Asha A, Kannan SM, Jayabalan V (2008) Optimization of clearance variation in selective assembly for components with multiple characteristics. Int J Adv Manuf Technol 38:1026-1044.

[11] Kumar MS, Kannan, SM (2007) Optimum manufacturing tolerance to selective assembly technique for different assembly specifications by using genetic algorithm. Int J Adv Manuf Technol 32:591-598.

[12] Raj MV, Sankar SS, Ponnambalam SG (2011) Minimizing clearance variations and surplus parts in multiple characteristic radial assembly through batch selective assembly. Int J Adv Manuf Technol 57:1199-1222.

[13] Akansel M, Emel E, Hacioglu V (2011) Optimal control of inventory accumulation in selective assembly process. Int J Adv Manuf Technol 56:729-742.

[14] Iyama T, Mizuno M, McKay KN, Yoshihara N, Nishikawa N (2013) Optimal strategies for corrective assembly approach applied to a high-quality relay production system. Computers in Industry 64:556-564.

[15] Fleischer J, Otter M, Beuke F (2014) Method to compensate production related deviations for the assembly of space-frame-structures. Production Engineering - Research and Development 8(1-2):207-216.

[16] Schmitt R, Niggemann C, Isermann M, Laass M, Matuschek N (2010) CognitionBased self-optimisation of an automotive rear-axle-drive production process. Journal of Machine Engineering 10(3):68-77.

[17] Arai T, Takeuchi K (1992) A Simulation System on Assembly Accuracy. CIRP Annals - Manufacturing Technology 41(1):37-40.

[18] Kayasa J, Herrmann C (2012) A Simulation Based Evaluation of Selective and Adaptive Production Systems (SAPS) Supported by Quality Strategies. Procedia CIRP 3:14-19.

[19] Peter M, Fleischer J (2014) Rotor Balancing by optimized Magnet Positioning during Algorithm-Controlled Assembly Process. 4th International Electric Drives Production Conference: 444-447.

[20] Engelhardt P, Reinhart G (2012) Approach for an RFID-based Situational Shop Floor Control. IEEEM 2012:444-448 